\title{
Narcissism predicts impulsive buying: phenotypic and genetic evidence
}

\author{
Huajian Cai', Yuanyuan Shi', Xiang Fang ${ }^{2}$ and Yu L. L. Luo ${ }^{1 *}$ \\ ${ }^{1}$ Key Laboratory of Behavioral Science, Institute of Psychology, Chinese Academy of Sciences, Beijing, China, ${ }^{2}$ Spears \\ School of Business, Oklahoma State University, Tulsa, OK, USA
}

Impulsive buying makes billions of dollars for retail businesses every year, particularly in an era of thriving e-commerce. Narcissism, characterized by impulsivity and materialism, may serve as a potential antecedent to impulsive buying. To test this hypothesis, two studies examined the relationship between narcissism and impulsive buying. In Study 1 , we surveyed an online sample and found that while adaptive narcissism was not correlated with impulsive buying, maladaptive narcissism was significantly predictive

OPEN ACCESS

Edited by:

Kimberly J. Saudino,

Boston University, USA

Reviewed by:

Michelle Anne Luke, University of Sussex, UK

Denis Bratko,

University of Zagreb, Croatia

*Correspondence: Yu L. L. LUO,

Key Laboratory of Behavioral Science, Institute of Psychology, Chinese Academy of Sciences, 16 Lincui Road, Beijing 100101, China luoy@psych.ac.cn

Specialty section:

This article was submitted to Personality and Social Psychology, a section of the journal Frontiers in Psychology

Received: 18 March 2015 Accepted: 15 June 2015 Published: 07 July 2015

Citation:

Cai $H$, Shi Y, Fang $X$ and Luo YLL (2015) Narcissism predicts impulsive buying: phenotypic and genetic evidence.

Front. Psychol. 6:881. doi: 10.3389/fpsyg.2015.00881 of the impulsive buying tendency. By investigating 304 twin pairs, Study 2 showed that global narcissism and its two components, adaptive and maladaptive narcissism, as well as the impulsive buying tendency were heritable. The study found, moreover, that the connections between global narcissism and impulsive buying, and between maladaptive narcissism and impulsive buying were genetically based. These findings not only establish a link between narcissism and impulsive buying but also help to identify the origins of the link. The present studies deepen our understanding of narcissism, impulsive buying, and their interrelationship.

\footnotetext{
Keywords: impulsive buying, narcissism, maladaptive narcissism, adaptive narcissism, behavior genetics, twin study
}

\section{Introduction}

On November 11, 2014, the so-called "double 11" date, consumers from 217 countries spent 57.1 billion Chinese Yuan (about US $\$ 9.3$ billion) on Alibaba, the largest Chinese online shopping site $^{1}$, standing in sharp contrast to the site's daily average purchase total. Why did so many consumers purchase so many items at this particular moment? Were the purchases planned in advance? We speculated that many people might have bought on impulse. Indeed, it is well documented that impulsive buying constitutes a large portion of daily purchases, particularly in this time of e-commerce (Kacen and Lee, 2002; Zhang and Shrum, 2009). In the U.S., for example, impulsive buying contributes US $\$ 4.2$ billion to annual sales (Mogelonsky, 1998) and accounts for 50\% of all mall purchases (Nichols et al., 2001). In the past half century, extensive research has been done on impulsive buying and established that impulsive buying could be either a spontaneous behavior

\footnotetext{
${ }^{1}$ This news was retrieved from the official site of the Alibaba Group: http://alibabagroup.com/cn/news/article?news=p141112
} [accessed December 01, 2014] 
triggered by situational factors, or a relatively stable tendency that varies across individuals (Rook, 1987; Rook and Fisher, 1995). In this research, we treated impulsive buying as a trait-like individual difference variable, and for the first time, investigated the relationship between narcissism and impulsive buying. We examined not only the phenotypic relationship between them, but also the genetic basis underlying this relationship.

\section{Impulsive Buying}

Impulsive buying refers to a spontaneous and compelling purchasing behavior, which is usually characterized by lack of reflection and deliberation on one hand and immediate satisfaction and pleasure on the other hand (Rook, 1987; Kacen and Lee, 2002). Impulsive consumption can be neutral or even positive (e.g., a spontaneous gift for a sick friend). Since it violates rationality principles of human economics and most people have limited financial recourses, impulsive buying, actually, is often associated with negative outcomes, such as financial problems and post-purchase dissatisfaction and regret (Rook and Hoch, 1985; Rook, 1987). Given its prevalence and potential influences, impulsive buying has received much attention from researchers in various areas, such as in behavioral economics (e.g., Underhill, 1999), marketing (e.g., Applebaum, 1951), and psychology (e.g., Rook, 1987).

Early research on this subject mainly focused on external factors, such as product features (Applebaum, 1951) and shopping environments (Stern, 1962) that may facilitate impulsive buying. Later, researchers realized that personal dispositional factors of consumers could play a more important role in impulsive buying (Rook and Hoch, 1985; Rook, 1987), just as Rook and Hoch (1985, p. 23) have articulated: "It is the individuals, not the products, who experience the impulse to consume." Most recently, researchers have identified many distinctive characteristics associated with impulsive buyers. Overall, they are more likely to be: (1) young rather than old and women rather than men (Dittmar et al., 1996; Wood and Ahuvia, 1998; Silvera et al., 2008); (2) high in hedonism, materialism, and individualism (Kacen and Lee, 2002; Zhang and Shrum, 2009); (3) high in extraversion, neuroticism, and impulsivity, but low in conscientiousness (Verplanken and Herabadi, 2001; Bratko et al., 2013; Lucas and Koff, 2014); and (4) low in selfcontrol and self-regulation (Baumeister, 2002; Vohs and Faber, 2007).

Extant research suggests that although impulsive buying may be triggered by various situational factors, it is also clearly associated with fundamental individual differences, including age, values, personality, and the ability to inhibit impulses. Researchers have already established the trait nature of the tendency for impulsive buying and developed corresponding measures (Rook and Fisher, 1995; Verplanken and Herabadi, 2001). Moreover, a recent twin study showed that the tendency for impulsive buying is heritable (Bratko et al., 2013). Following this individual difference perspective, we examined the relationship between the tendency for impulsive buying and narcissism as well as its possible genetic bases.

\section{Narcissism and Impulsive Buying}

Narcissism $^{2}$ is typically characterized by a grandiose self-view. Narcissists ${ }^{3}$ are individuals who consider themselves to be special, entitled, superior to others, and at the same time, try to make others believe in this aggrandized self-image. Therefore, validating and promoting the inflated self constitutes an endless desire for narcissists. Habitually, they are ready to use all means to protect and elevate their excessively positive self (Morf and Rhodewalt, 2001). Among the various ways in which narcissists self-enhance, making purchases is an important one. Material possessions can promote a person's apparent status, sustain a grandiose self-image, and influence others' opinions of the individual (Sedikides et al., 2007; Cisek et al., 2008). Display of wealth can be an effective means to breed a favorable impression (Christopher and Schlenker, 2000). Overwhelmingly, narcissistic people exhibit high materialism (Rose, 2007) and strong aspirations for wealth, fame, and a positive image (Kasser and Ryan, 1996; Roberts and Robins, 2000), and tend to enhance their self-image by purchasing products, especially symbolic, exclusive, and personalized ones (Cisek et al., 2008; Lee et al., 2013).

In this article, we argue that narcissists are not only more likely to purchase, but also more likely to purchase on impulse. There are good reasons for us to hypothesize this tendency. First, narcissism is characterized by impulsivity (Raskin and Terry, 1988), the primary drive behind impulsive buying (Rook, 1987). A meta-analysis of 23 correlations between impulsivity and narcissism generated a mean effect size of $r=0.41$ (Vazire and Funder, 2006; but see Miller et al., 2009). Second, impulsive buyers share several characteristics in common with narcissists, such as pursuing a positive self-identity (Dittmar et al., 1995, 1996; Dittmar and Drury, 2000), materialism, and individualism (Kacen and Lee, 2002; Zhang and Shrum, 2009). Third, narcissistic people are inclined to engage in compulsive buying, a pathological form of consumer behavior resembling impulsive buying in high materialism and low impulse control (O'Guinn and Faber, 1989; Rose, 2007; Ridgway et al., 2008). Consistent with these factors, empirical studies found that people with highly positive self-views liked to engage in impulsive buying to enhance their self-image (Cash and Cash, 1982) and people who exaggerated their attractiveness exhibited stronger impulsive buying tendencies (Lucas and Koff, 2014). To test the hypothesis that narcissism may predict the tendency for impulsive buying, we examined the relationship of impulsive buying with overall narcissism first and thereafter with its two components: adaptive narcissism and maladaptive narcissism.

\section{Adaptive Narcissism vs. Maladaptive Narcissism}

In most studies among normal individuals, narcissism has been assessed by the Narcissistic Personality Inventory (NPI), which

\footnotetext{
${ }^{2}$ Throughout the article, "narcissism" refers to individual difference that is continuous within normal populations, i.e., subclinical narcissism.

${ }^{3}$ For the convenience of terminology, we use "narcissist" to refer a normal person exhibiting relatively high subclinical narcissism.
} 
includes seven subscales of authority, entitlement, exhibitionism, exploitation, self-sufficiency, superiority, and vanity (Raskin and Terry, 1988). Yet in most cases, narcissism has been limited to being treated as a whole and has assumed to be maladaptive (Emmons, 1987; Hepper et al., 2013). In recent years, however, increasing evidence suggests that some components of narcissism, such as authority/leadership and self-sufficiency, are adaptive but some other components, such as exploitativeness, entitlement, and exhibitionism, are maladaptive (Watson and Biderman, 1993; Ackerman et al., 2011).

Thus far, research has established distinctions between adaptive and maladaptive narcissism. High adaptive narcissism has been shown to be associated with high conscientiousness, subjective well-being, self-esteem, assertiveness, self-confidence, and self-control, whereas high maladaptive narcissism is associated with high neuroticism, anxiety, social anxiety, depression, social maladjustment, Machiavellianism, impulsive antisociality, delinquency, aggression and low empathy, conscientiousness, and subjective well-being (Emmons, 1984; Raskin and Terry, 1988; Watson and Biderman, 1993; Washburn et al., 2004; Barry et al., 2007; Ackerman et al., 2011; Hepper et al., 2014). Based on these existing findings, we can see that it is maladaptive narcissism rather than adaptive narcissism that is associated with various negative outcomes. Therefore, we not only examined the relationship between impulsive buying and overall narcissism, but also the relationship between its two components, adaptive and maladaptive narcissism, and impulsive buying. We expected that maladaptive narcissism, instead of adaptive narcissism, would be predictive of impulsive buying.

\section{Heritability of Narcissism and Impulsive Buying}

Behavioral genetic research has established that individual differences in narcissism are partly determined by genetic factors in both eastern and western cultures (Vernon et al., 2008; Luo et al., 2014). In addition, the connections between narcissism and personality traits, such as extraversion, openness, and conscientiousness, are largely due to common genetic influences (Vernon et al., 2008). Regarding impulsive buying, only one twin study has been done to date, its results showing that both impulsive buying and its associations with impulsivity, neuroticism, and extraversion are heritable (Bratko et al., 2013). No twin study, however, has simultaneously examined narcissism and impulsive buying, which constitutes a primary impetus for our work.

\section{Overview}

In summary, we aimed to examine the relationship between narcissism and impulsive buying. We conducted two studies. While Study 1 capitalized on an online sample, Study 2 used a twin sample, which allowed us to investigate the genetic foundation of possible relationships between narcissism and impulsive buying.

\section{Study 1}

\section{Method \\ Participants}

One-hundred and twelve individuals (all Chinese, 47\% male) recruited through a Chinese witkey website (i.e., www.zhubajie. com) participated in the online study. Their ages ranged from 17 to 38 years $(M=25.06, S D=3.88)$. Every participant received CNY5 (about US \$0.82) in compensation. The Ethics Committee of the Institute of Psychology, Chinese Academy of Sciences provided approval for the study. Additionally, we obtained written informed consent from all participants prior to commencing the test.

\section{Measures}

Narcissism was measured with the Narcissistic Personality Inventory (NPI; Raskin and Terry, 1988). The 40-item NPI has been successfully used in Chinese samples (Cai et al., 2012). Each item includes a pair of statements, one narcissistic and the other non-narcissistic as in the example, "I prefer to blend in with the crowd" (non-narcissistic statement) vs. "I like to be the center of attention" (narcissistic statement). For each item, participants indicated whether the narcissistic or non-narcissistic statement better described them. We coded the narcissistic statement choice as 1 and the non-narcissistic statement choice as 0 . The internal consistency for the whole scale was desirable $(\alpha=0.82)$. We calculated scores for adaptive and maladaptive narcissism based on previous decomposition of the NPI (Hepper et al., 2014). Specifically, 14 items reflecting authority and self-sufficiency were totaled to assess adaptive narcissism $(\alpha=0.67)$, and 18 items concerning entitlement, exploitativeness, and exhibitionism were added up to index maladaptive narcissism $(\alpha=0.61)$. The moderate internal consistencies of the subscales are congruent with past research, and in any case, have shown good construct validity and test-retest reliability (Barry et al., 2007; Barry and Malkin, 2010; Hepper et al., 2014).

Impulsive buying was measured with the Impulsive Buying Scale (Rook and Fisher, 1995). The scale includes nine items, such as the statement, "I often buy things spontaneously." The participant indicated his or her agreement with these statements on a 7 -point Likert scale $(1=$ completely disagree, $7=$ completely agree). The scale was translated into Chinese and back-translation was used to ensure language equivalence. The scale was internally consistent $(\alpha=0.86)$, and a mean score was calculated for each participant.

\section{Results}

For the overall NPI score $(M=12.03, S D=6.20)$, its relationship with the tendency for impulsive buying $(M=3.64, S D=1.2)$ was positive but not significant $(r=0.14, p=0.157)$. For the two subcomponents of narcissism, while the maladaptive narcissism score $(M=5.64, S D=2.95)$ was positively correlated with impulsive buying $(r=0.20, p=0.034)$, the adaptive narcissism was not $(M=4.15, S D=2.63 ; r=0.03, p=0.784)$. In summary, although we failed to find a significant relation between overall narcissism and impulsive buying, we did found that maladaptive narcissism was significantly predictive. 


\section{Study 2}

\section{Method \\ Participants}

One-hundred and fifty-two monozygotic (MZ) and one-hundred and fifty-two dizygotic (DZ; 94 same-sex, 58 opposite-sex) twin pairs sampled from the Beijing Twin Study (BeTwiSt) participated in the study. Twins in the BeTwiSt are sociodemographically representative of adolescents from Beijing, China (Chen et al., 2013). The ages of the twins in our sample ranged from 15 to 27 years ( $M=18.29, S D=1.96 ; 56 \%$ female). For $95 \%$ of the twin-pairs, we used DNA testing to determine zygosity, with classification accuracy approaching $100 \%$; for the remaining $5 \%$, we established zygosity by combining parentreports and children's self-reports on co-twin physical similarity and frequency of confusion, which reached a $90.6 \%$ accuracy rating (Chen et al., 2013). Thirty-five twin pairs did not complete the measure for impulsive buying. The data for one sibling of a DZ twin pair was missing and thus excluded in all analyses. The Ethics Committee of the Institute of Psychology, Chinese Academy of Sciences, provided approval for the study. Additionally, we obtained written informed consent from all participants and their parents prior to commencing the test.

\section{Measures}

The measures for narcissism and impulsive buying were the same as those in Study 1. The internal consistency for the entire NPI, adaptive narcissism, maladaptive narcissism, and impulsive buying, were all acceptable $(\alpha=0.81,0.63,0.66,0.89$, respectively).

\section{Genetic Analysis}

We can estimate the additive genetic (A), shared environmental (C), and non-shared environmental (E) contributions to variance within a trait and covariance between traits by comparing the resemblance of $\mathrm{MZ}$ and $\mathrm{DZ}$ twin pairs for observed trait(s). MZ twins are $100 \%$ genetically identical, whereas DZ twins are $50 \%$ identical on average for additive genetic effects. The proportion of the variance of a trait, or the covariance between traits, explained by additive genetic effect, is referred as "heritability." A shared environment increases the similarity of twins raised in the same family. A non-shared environment is unique to each individual, which also includes measurement error.

To estimate genetic and environmental effects, we employed univariate, bivariate, and multivariate models implemented in the OpenMx library (Boker et al., 2012) within the $\mathrm{R}$ statistical computing environment ( $\mathrm{R}$ Development Core Team, 2012). First, the univariate model decomposed the variances of maladaptive narcissism and impulsive buying, respectively, into genetic (A) and environmental (C, E) effects. We examined the full ACE model first. Sub-models nested under the full model were also tested by systematically removing one component of variance.

For the bivariate analysis of overall narcissism and impulsive buying, we used a correlated factors model based on Cholesky decomposition (Loehlin, 1996). Cholesky decomposition is similar to hierarchical regression analyses in non-genetic studies, through which the independent contribution of predictors entered later is assessed after controlling for the predictors entered earlier (Loehlin, 1996). Bivariate analysis allows the covariance of two traits to be partitioned into covariance that is due to additive genetic factors, common environmental factors and unique environmental factors. In this model, each variable is separately decomposed into its genetic, shared and nonshared environmental components at the same time that the correlations of these across variables are estimated (Figure 1A). A genetic correlation $\left(r_{\mathrm{g}}\right)$ indicates the extent to which genetic influences on one trait overlap with those on the second trait (regardless of their individual heritabilities), just as in shared $\left(r_{\mathrm{c}}\right)$, and non-shared $\left(r_{\mathrm{e}}\right)$ environmental correlations. Similar to the univariate analysis, the full ACE model and the sub-models were systematically tested.
A

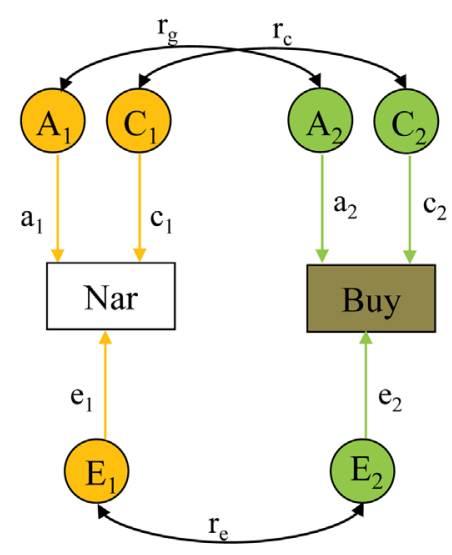

FIGURE 1 | Bivariate model-fitting for narcissism (Nar) and impulsive buying (Buy). (A) Path diagram illustrating the bivariate model-fitting. (B) The best-fitting bivariate model. Measured variables are depicted in rectangles. Latent factors $\mathrm{A}$ (additive genetic factor), $\mathrm{C}$ (shared environmental factor), and $\mathrm{E}$
B

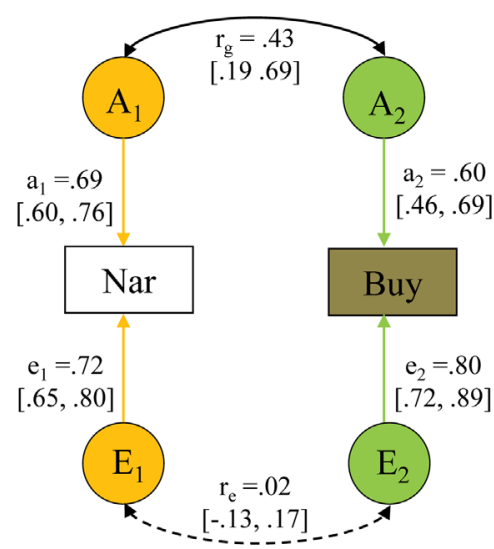

(non-shared environmental factor) are presented in circles. $r_{g}$, genetic correlation; $r_{\mathrm{c}}$, shared environmental correlation; $r_{\mathrm{e}}$, non-shared environmental correlation. All path estimates (95\% confidence intervals) are standardized but unsquared. The non-significant path is represented by a dashed line. 
Finally, for the multivariate analysis of adaptive narcissism, maladaptive narcissism, and impulsive buying, we used Cholesky decomposition. The multivariate model parameterized the variances for and the covariances among adaptive narcissism, maladaptive narcissism, and impulsive buying into three groups of genetic and environmental effects (Figure 2). $A_{1}, C_{1}$, and $\mathrm{E}_{1}$ represent genetic, shared environmental, and non-shared environmental influences common to all measures; $A_{2}, C_{2}$, and $\mathrm{E}_{2}$ represent influences common to the second and the last variables in the model; and $A_{3}, C_{3}$, and $E_{3}$ represent influences unique to the last variable. Notably, $A_{1}, C_{1}$, and $\mathrm{E}_{1}$ also include influences specific to the first variable in the model, whereas $A_{2}, C_{2}$, and $E_{2}$ also include influences specific to the second variable in the model. We tested two Cholesky models. As personality theories typically hypothesize traits as causes of behaviors, rather than the reverse (Allport, 1937; McCrae and Costa, 1999), we entered impulsive buying last in both models. In one model (Figure 2A), we entered adaptive narcissism first and maladaptive narcissism thereafter, so that we could examine the specific genetic and environmental influences from maladaptive narcissism to impulsive buying. In the other model (Figure 2B), we entered maladaptive narcissism first and adaptive narcissism second, so that we could also examine the specific genetic and environmental influences from adaptive narcissism to impulsive buying. The two models operate in the same way except that the order of entering the variables is reversed. From the model paths, we can estimate genetic, shared, and non-shared environmental effects on adaptive narcissism, maladaptive narcissism, and impulsive buying. We also can estimate correlations between these effects (i.e., genetic, shared, and non-shared environmental correlations) based on the model paths. As with the previous analyses, the full ACE model and the sub-models were systematically tested.

We used the change in chi-square $\left(\chi^{2}\right)$ and Akaike's Information Criterion (AIC; Akaike, 1987) as model fit indices. A lower AIC value indicates better fit. To compare a submodel with the full model, a significant chi-square difference suggests that the nested model performs worse than the full model, resulting in retaining the full model; otherwise, the nested model with fewer parameters fits better in terms of parsimony (Bollen, 1989; Kline, 1998). Hence, the better-fit model receives due consideration (Kline, 1998).

\section{Results}

\section{Descriptive Statistics}

The overall NPI score $(M=13.64, S D=6.05)$ was modestly related to impulsive buying $(r=0.23, p<0.001)$. As in Study 1 , maladaptive narcissism $(M=6.25, S D=3.10)$ was significantly correlated with impulsive buying $(M=3.18, S D=1.28 ; r=0.29$, $p<0.001)$, but adaptive narcissism $(M=4.90, S D=2.52)$ was not $(r=0.09, p=0.147)$. Consistent with Study 1, it was maladaptive but not adaptive narcissism that predicted impulsive buying, providing convergent evidence for our expectations. Inconsistent with Study 1, however, the relationship between overall narcissism and impulsive buying was significant.

\section{Univariate Genetic Analysis}

Because twins are perfectly correlated for age and for gender when of the same gender, variations associated with age or gender would inflate the correlation between twins. Following standard procedure, all measures were corrected for age and gender effects using multiple regression and standardized residuals were saved for genetic analyses (McGue and Bouchard, 1984).

\section{Impulsive buying}

On impulsive buying, the MZ twin correlation (0.49) was higher than the DZ correlation (0.33; Table 1), suggesting that it is heritable. Next, we examined heritability by fitting a series

TABLE 1 | Twin intraclass correlations (ICC).

\begin{tabular}{lllll}
\hline Measure & $\mathbf{I C C}_{\mathbf{M Z}}$ & $\mathbf{N}_{\mathbf{M Z}}$ & $\mathbf{I C C}_{\mathbf{D Z}}$ & $\mathbf{N}_{\mathbf{D Z}}$ \\
\hline Maladaptive narcissism & $0.66(0.53-0.75)$ & 152 & $0.15(-0.18-0.38)$ & 151 \\
Adaptive narcissism & $0.55(0.38-0.68)$ & 152 & $0.31(0.04-0.50)$ & 149 \\
Impulsive buying & $0.49(0.27-0.64)$ & 127 & $0.33(0.06-0.52)$ & 142 \\
\hline
\end{tabular}

MZ, monozygotic twins; DZ, dizygotic twins. 95\% confidence intervals are in parentheses.
A

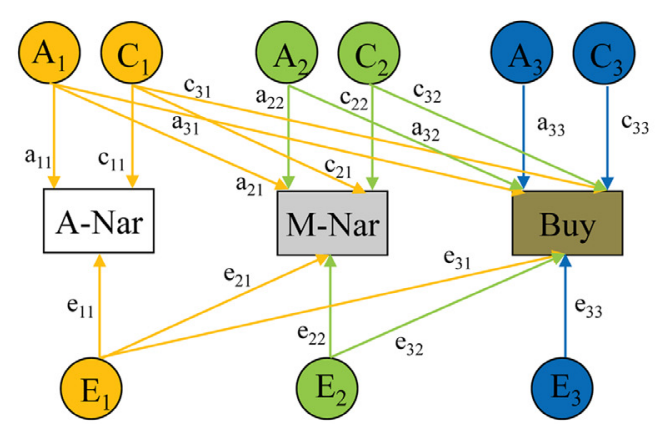

FIGURE 2 | Path diagram illustrating the multivariate model-fitting for maladaptive narcissism (M-Nar), adaptive narcissism (A-Nar), and impulsive buying (Buy). (A) The model from adaptive narcissism to maladaptive narcissism and impulsive buying. (B) The model from maladaptive
B

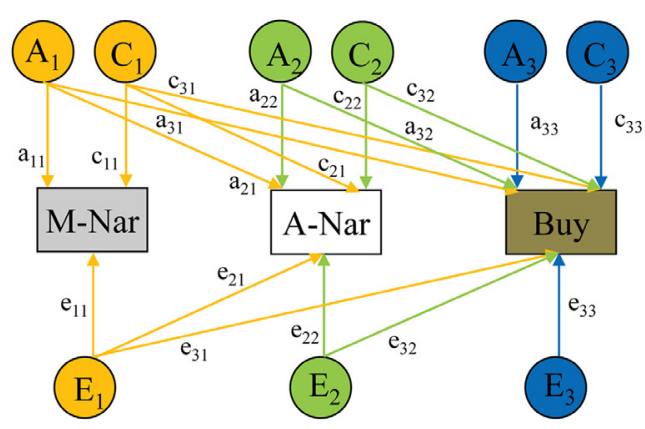

narcissism to adaptive narcissism and impulsive buying. Measured variables are depicted in rectangles. Latent factors A (additive genetic factor), C (shared environmental factor), and $E$ (non-shared environmental factor) are presented in circles. 


\begin{tabular}{|c|c|c|c|c|c|c|c|c|c|c|}
\hline \multirow[b]{2}{*}{ Measure } & \multirow[b]{2}{*}{ Model } & \multirow[b]{2}{*}{$-2 L L$} & \multirow[b]{2}{*}{$d f$} & \multirow[b]{2}{*}{ AIC } & \multicolumn{3}{|c|}{ Change from full model } & \multirow[b]{2}{*}{$a^{2}$} & \multirow[b]{2}{*}{$c^{2}$} & \multirow[b]{2}{*}{$\mathrm{e}^{2}$} \\
\hline & & & & & $\Delta \chi^{2}$ & $\Delta d f$ & $p$ & & & \\
\hline \multirow[t]{4}{*}{ Impulsive buying } & ACE & 1503.60 & 534 & 435.60 & & & & $0.27(0.00-0.47)$ & 0.07 (0.00-0.35) & $0.67(0.53-0.83)$ \\
\hline & $\underline{A E}$ & 1503.74 & 535 & 433.74 & 0.14 & 1 & 0.708 & $0.34(0.20-0.47)$ & & $0.66(0.53-0.80)$ \\
\hline & CE & 1505.06 & 535 & 435.06 & 1.46 & 1 & 0.227 & & $0.26(0.15-0.37)$ & $0.74(0.63-0.85)$ \\
\hline & $E$ & 1524.03 & 536 & 452.03 & 20.43 & 2 & 0.000 & & & $1.00(1.00-1.00)$ \\
\hline \multirow[t]{4}{*}{ Maladaptive narcissism } & ACE & 1677.244 & 602 & 473.24 & & & & $0.44(0.25-0.55)$ & $0.00(0.00-0.14)$ & $0.56(0.45-0.69)$ \\
\hline & $\underline{A E}$ & 1677.244 & 603 & 471.24 & 0.00 & 1 & 1.000 & $0.44(0.31-0.55)$ & & $0.56(0.45-0.69)$ \\
\hline & CE & 1689.181 & 603 & 483.18 & 11.94 & 1 & 0.001 & & $0.29(0.19-0.39)$ & $0.71(0.61-0.81)$ \\
\hline & $E$ & 1716.495 & 604 & 508.50 & 39.25 & 2 & 0.000 & & & $1.00(1.00-1.00)$ \\
\hline \multirow[t]{4}{*}{ Adaptive narcissism } & ACE & 1651.486 & 599 & 453.49 & & & & $0.37(0.00-0.49)$ & $0.00(0.00-0.32)$ & $0.63(0.51-0.77)$ \\
\hline & $\underline{A E}$ & 1651.486 & 600 & 451.49 & 0.00 & 1 & 1.000 & $0.37(0.24-0.49)$ & & $0.63(0.51-0.76)$ \\
\hline & CE & 1654.94 & 600 & 454.94 & 3.45 & 1 & 0.063 & & $0.28(0.18-0.38)$ & $0.72(0.62-0.82)$ \\
\hline & $E$ & 1680.057 & 601 & 478.06 & 28.57 & 2 & 0.000 & & & $1.00(1.00-1.00)$ \\
\hline
\end{tabular}

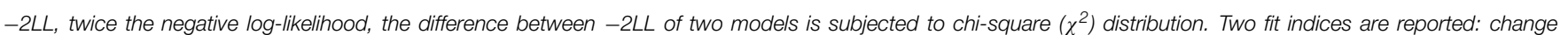

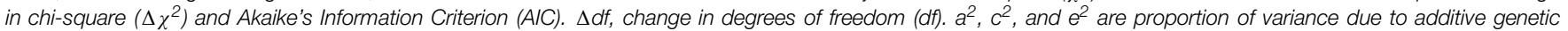

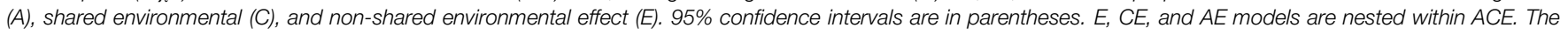
best-fit model is underlined.

of univariate models (Table 2). The full ACE model showed a moderate heritability of $27 \%$ with shared (7\%) and nonshared $(67 \%)$ environments accounting for the rest of the individual difference. Removing either $\mathrm{C}$ (AE model, $p=0.708$ ) or A (CE model, $p=0.227$ ) did not significantly change the model fit. But the simultaneous exclusion of $\mathrm{A}$ and $\mathrm{C}$ (E model, $p<0.001$ ) decreased the model fit. In addition, the AE model displayed the lowest AIC value within the full model (435.60) and all sub-models (AE: 433.74, CE: 435.06, E: 452.03) and was chosen for this reason (Bollen, 1989; Kline, 1998). According to the $\mathrm{AE}$ model, genetic and nonshared environmental factors accounted for 34 and $66 \%$ of the individual differences in impulsive buying, respectively. The results were comparable to previous findings (Bratko et al., 2013).

\section{Overall narcissism}

The MZ twin correlation was higher than that of $\mathrm{DZ}$ twins $(\mathrm{MZ}=0.66, \mathrm{DZ}=0.36)$. The full ACE model found substantial genetic (47\%) and non-shared environmental effects (53\%), but no significant shared environmental effect $(0 \%)$. Since the AE model fitted the data as well as the ACE model $(p=1.000)$, the AE model was preferable according to the parsimony principle (Bollen, 1989; Kline, 1998). Based on the AE model, overall narcissism was moderately heritable, with substantial influence drawn from non-shared environments ${ }^{4}$.

\section{Maladaptive narcissism}

For maladaptive narcissism, MZ twins (0.66) resembled each other two times more than DZ twins did (0.15; Table 1), suggesting significant genetic influence and trivial shared environmental influence. A series of univariate model-fitting

${ }^{4}$ These results were reported in Luo et al. (2014).

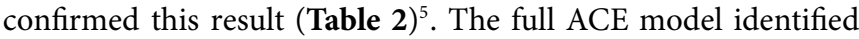
a heritability of $44 \%$ and a non-shared environmental effect of $56 \%$, with zero contribution from shared environment. The removal of the $\mathrm{C}$ component did not decrease the model fit $(p=1.000)$. But dropping A (CE model, $p=0.001)$ or both A and $C$ (E model, $p<0.001)$ significantly reduced the model fit. Hence, the AE model was preferable (Bollen, 1989; Kline, 1998). In the AE model, the estimates for the genetic and non-shared environmental influences were the same as those in the ACE model.

\section{Adaptive narcissism}

On adaptive narcissism, MZ twins (0.55) resembled each other more than DZ twins did (0.31; Table 1), which suggested genetic influences. Subsequently, we employed univariate model-fitting to estimate the genetic and environmental contributions to the individual differences in adaptive narcissism (Table 2). Based on the results from the ACE model, 37\% of individual differences in adaptive narcissism could be explained by genetic factors, with the other $63 \%$ attributed to non-shared environment and an estimate of zero for shared environment. The AE model fitted the data as well as the full ACE model $(p=1.000)$, whereas the $\mathrm{CE}(p=0.063)$ and $\mathrm{E}(p<0.001)$ models decreased the fitness at least to a marginal extent. Following the parsimony principle (Kline, 1998), the AE models provided the best account of the variances in adaptive narcissism.

\footnotetext{
${ }^{5}$ Although MZ correlations are more than twice as large as DZ correlations for maladaptive narcissism, we did not estimate the dominance genetic effect (ADE model) due to the lack of power arising from our relatively small sample (Martin et al., 1978). Another reason we did not test the ADE model is that previous twin studies of narcissism, all of which were measured with the NPI as we did, found that genetic effects on narcissism are additive rather than dominant (Vernon et al., 2008; Luo et al., 2014). In particular, Luo et al. (2014) and the present study are based on the same sample as well as the same measure of narcissism, i.e., NPI. As for the DE model, it was never tested given that a model with dominant genetic variance that lacks additive genetic variance is biologically implausible (Neale and Cardon, 1992).
} 
TABLE 3 | Bivariate model-fitting of impulsive buying and overall narcissism.

\begin{tabular}{lllllll}
\hline & & & & \multicolumn{3}{c}{ Change from full model } \\
\cline { 5 - 7 } Model & $\mathbf{- 2 L L}$ & $\boldsymbol{d} \boldsymbol{f}$ & AIC & $\boldsymbol{\Delta} \boldsymbol{\chi}^{\mathbf{2}}$ & $\boldsymbol{\Delta} \boldsymbol{d f}$ & $\boldsymbol{p}$ \\
\hline ACE & 3137.76 & 1132 & 873.76 & & & \\
AE & 3141.43 & 1135 & 871.43 & 3.66 & 3 & 0.301 \\
CE & 3150.89 & 1135 & 880.89 & 13.13 & 3 & 0.000 \\
E & 3210.02 & 1138 & 934.02 & 72.26 & 6 & 0.000 \\
\hline
\end{tabular}

-2LL, twice the negative log-likelihood; AIC, Akaike's Information Criterion; $\Delta \chi^{2}$, change in chi-square; $\Delta d f$, change in degrees of freedom (df); $A$, additive genetic effects; $C$, shared environmental effects; $E$, non-shared environmental effects. $E$, $C E$, and AE models are nested within the ACE model. The best fitting model is underlined.

\section{Bivariate Genetic Analysis of Overall Narcissism and Impulsive Buying}

We tested the full ACE model first and thereafter the AE, CE, and $\mathrm{E}$ models (Table 3). Compared with the full model, the $\mathrm{AE}$ model fitted the data equally well $(p=0.301)$. But the CE and E models ( $p s<0.001)$ were significantly worse. In line with the univariate model-fitting, the AE model was optimal (Figure 1B). The AE model identified a medium genetic correlation $(0.44,95 \%$ CI: $0.19,0.69)$, but a non-significant non-shared environmental correlation $(0.02,95 \%$ CI: $-0.13,0.17)$ between narcissism and impulsive buying. This outcome suggested that narcissism and impulsive buying share genetic sources to a considerable extent, whereas the non-shared environment underlying them is largely different.

\section{Multivariate Genetic Analysis of Adaptive, Maladaptive Narcissism, and Impulsive Buying}

Two multivariate models were applied to analyze the genetic and environmental influences from adaptive narcissism to maladaptive narcissism and impulsive buying (Figure 2A), and from maladaptive narcissism to adaptive narcissism and impulsive buying (Figure 2B), respectively. For each model, we tested the full ACE model first and thereafter the AE, CE, and E models (Table 4). Compared with the full model, the AE model fitted the data equally well $(p=0.861)$. But the $\mathrm{CE}$ and $\mathrm{E}$ models were significantly worse $(p s<0.01)$. In line with the univariate model-fitting, the AE model was optimal (Figure 3). It is worth noting that the two chosen AE models fitted the data equally well because they functioned in the same way on the same data, with the exception of the order of variables (i.e., adaptive and maladaptive narcissism).

As seen in Figure 3, the genetic effect on impulsive buying was composed of three parts. One part was caused by genetic influences common to all variables $\left(A_{1}\right)$, another part by genetic influences shared with maladaptive/adaptive narcissism $\left(A_{2}\right)$, and the remaining part by unique genetic influences $\left(A_{3}\right)$. Comparing Figure 3A and Figure 3B, we found that, (1) after controlling for adaptive narcissism (Figure 3A), genetic factors (i.e., $\mathrm{A}_{2}$ ) influencing maladaptive narcissism also exerted significant influence on impulsive buying $\left(\mathrm{a}_{32}=0.25,95 \%\right.$ CI: $0.04,0.44)$; (2) after controlling for maladaptive narcissism
TABLE 4 | Multivariate model-fitting of impulsive buying, adaptive narcissism, and maladaptive narcissism.

\begin{tabular}{|c|c|c|c|c|c|c|}
\hline \multirow[b]{2}{*}{ Model } & \multirow[b]{2}{*}{$-2 L L$} & \multirow[b]{2}{*}{$d f$} & \multirow[b]{2}{*}{ AIC } & \multicolumn{3}{|c|}{ Change from full model } \\
\hline & & & & $\Delta \chi^{2}$ & $\Delta d f$ & $p$ \\
\hline \multicolumn{7}{|c|}{ Adaptive narcissism $\rightarrow$ Maladaptive narcissism $\rightarrow$ Impulsive buying (Figure 3A) } \\
\hline ACE & 4629.18 & 1726 & 1177.18 & & & \\
\hline$\underline{A E}$ & 4631.75 & 1732 & 1167.75 & 2.57 & 6 & 0.861 \\
\hline CE & 4647.51 & 1732 & 1183.51 & 18.33 & 6 & 0.005 \\
\hline$E$ & 4707.73 & 1738 & 1231.73 & 78.55 & 12 & 0.000 \\
\hline \multicolumn{7}{|c|}{ Maladaptive narcissism $\rightarrow$ Adaptive narcissism $\rightarrow$ Impulsive buying (Figure 3B) } \\
\hline ACE & 4629.18 & 1726 & 1177.18 & & & \\
\hline$\underline{\mathrm{AE}}$ & 4631.75 & 1732 & 1167.75 & 2.57 & 6 & 0.861 \\
\hline CE & 4647.51 & 1732 & 1183.51 & 18.33 & 6 & 0.005 \\
\hline E & 4707.73 & 1738 & 1231.73 & 78.55 & 12 & 0.000 \\
\hline
\end{tabular}

-2LL, twice the negative log-likelihood; AIC, Akaike's Information Criterion; $\Delta \chi^{2}$, change in chi-square; $\Delta d f$, change in degrees of freedom (df); $A$, additive genetic effects; $C$, shared environmental effects; $E$, non-shared environmental effects. $E$, $C E$, and AE models are nested within the ACE model. The best fitting model is underlined. Because the two sets of models functioned in the same way, except the order of variables (i.e., adaptive and maladaptive narcissism), on the same data, they provided the same fit to the data.

(Figure 3B), however, genetic factors (i.e., $A_{2}$ ) influencing adaptive narcissism imparted trivial influence on impulsive buying $\left(\mathrm{a}_{32}=-0.02,95 \% \mathrm{CI}:-0.24,0.18\right)$. Similarly, the nonshared environmental effect also included three parts. But only influence from the unique factor $\left(\mathrm{E}_{3}\right)$ were significant $\left(\mathrm{e}_{33}=.80\right.$, $95 \%$ CI: $0.72,0.88)$. Influences from the two shared factors $\left(\mathrm{E}_{1}\right.$, $\mathrm{E}_{2}$ ) were limited ( $\mathrm{e}_{31}$ and $\mathrm{e}_{32}$ ranged from -0.05 to $0.08,95 \%$ CIs included zero).

In addition, between maladaptive narcissism and impulsive buying, the multivariate analysis identified a medium genetic correlation $\left(r_{\mathrm{g}}=0.53,95 \%\right.$ CI: $\left.0.27,0.78\right)$, but a trivial nonshared environmental correlation $\left(r_{\mathrm{e}}=0.08,95 \% \mathrm{CI}:-0.08\right.$, $0.23)$. This outcome suggested that maladaptive narcissism and impulsive buying share genetic sources to a considerable extent, whereas the non-shared environment underlying them is largely different. Regarding adaptive narcissism and impulsive buying, the analysis showed that there was a modest genetic correlation between them $\left(r_{\mathrm{g}}=0.33,95 \% \mathrm{CI}: 0.04,0.64\right)$. Since we identified no specific genetic influences from adaptive narcissism to impulsive buying after maladaptive narcissism was controlled, the genetic overlap between adaptive narcissism and impulsive buying was largely attributed to genetic influences shared with maladaptive narcissism (i.e., $A_{1}$ ). We also found a minimal nonshared environmental correlation between adaptive narcissism and impulsive buying $(-0.03,95 \% \mathrm{CI}:-0.17,0.12)$. This finding indicated that non-shared environments underlying them are mostly different.

In summary, we found that the tendency for impulsive buying and overall narcissism as well as their association were heritable. As for the two components of narcissism, adaptive and maladaptive narcissism, they both were also heritable. Again, maladaptive narcissism but not adaptive narcissism predicted impulsive buying. Moreover, the association between 
A

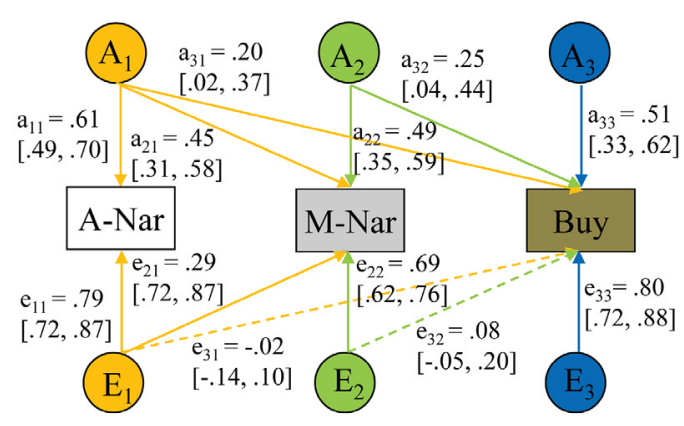

FIGURE 3|The best-fitting multivariate model for maladaptive narcissism (M-Nar), adaptive narcissism (A-Nar), and impulsive buying (Buy). (A) The model from adaptive narcissism to maladaptive narcissism and impulsive buying. (B) The model from maladaptive narcissism to adaptive narcissism and impulsive buying.
B

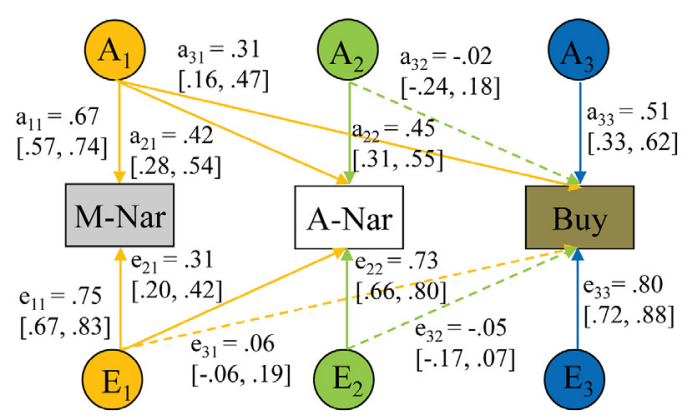

Measured variables are depicted in rectangles. Latent factors $A$ (additive genetic factor), $\mathrm{C}$ (shared environmental factor), and $\mathrm{E}$ (non-shared environmental factor) are presented in circles. All path estimates (95\% confidence intervals) are standardized but unsquared. The non-significant path is represented by a dashed line. maladaptive narcissism and impulsive buying had some genetic basis.

\section{Discussion}

Impulsive buying is pervasive. This behavior can be attributed to situational triggers, personal dispositions, or both. In this research, we treated impulsive buying as a trait-like disposition and examined whether narcissism may predispose some individuals to be more likely to conduct impulsive buying. We found that the correlation between global narcissism and impulsive buying is significant, whereby individuals with high narcissism are more likely to engage in impulsive buying ${ }^{6}$. For two components of narcissism, we found that high maladaptive narcissism rather than high adaptive narcissism predicts high impulsive buying. Moreover, we learned that the relationship between global narcissism and impulsive buying as well as between maladaptive narcissism and impulsive buying have genetic bases. These results have important implications.

Impulsivity gives rise to a substantial portion of our daily purchases. Previous studies have revealed many predictors of impulsive buying, including age (Wood and Ahuvia, 1998), gender (Dittmar et al., 1996), culture (Kacen and Lee, 2002), affect (Verplanken et al., 2005), the Big Five Personality traits (Verplanken and Herabadi, 2001), self-control (Vohs and Faber, 2007), and others. We identified a novel predictor of impulsive buying, that is, narcissism, thus confirming our hypothesis. This finding matches previous findings that narcissists are impulsive (Vazire and Funder, 2006; but see Miller et al., 2009) and materialistic (Kasser and Ryan, 1996; Roberts and Robins, 2000) and that those individuals with a highly positive self-view are more likely to buy on impulse (Cash and Cash, 1982). As

\footnotetext{
${ }^{6}$ The correlation between global narcissism and impulsive buying in Study 1 is positive but not significant. We conducted a meta-analysis to combine the correlation from Study $1(r=0.14, N=112)$ and Study $2(r=0.23, N=304)$. The weighted mean correlation across two studies is significant $(r=0.21,95 \% \mathrm{CI}$ : $0.12,0.29)$.
}

expected, maladaptive narcissism rather than adaptive narcissism is particularly predictive of impulsive buying. This result is in line with a large body of pre-existing findings whereby a negative outcome is usually associated with maladaptive narcissism rather than adaptive narcissism and thus adds to the mounting evidence that adaptive and maladaptive narcissism are distinct and have different functions (Emmons, 1984; Raskin and Terry, 1988; Watson and Biderman, 1993; Washburn et al., 2004; Barry et al., 2007; Ackerman et al., 2011; Hepper et al., 2014). In summary, our findings contribute novel detail to the portrait of an impulsive buyer.

More important, the discovery of the link between impulsive buying and narcissism is strengthened by the twin study. Past twin studies have demonstrated that both narcissism and the tendency for impulsive buying are heritable (Vernon et al., 2008; Bratko et al., 2013; Luo et al., 2014). We successfully replicated these findings in a twin sample from Beijing, China, with genetic factors accounting for $34 \%$ of the variations for impulsive buying, and 47,37 , and $44 \%$ of the variations for global narcissism, adaptive and maladaptive narcissism, respectively. We found significant genetic correlation between global narcissism and impulsive buying. As for narcissism's two subcomponents, we did not find a significant relationship between adaptive narcissism and impulsive buying. We did discover, however, a significant relationship between maladaptive narcissism and impulsive buying, which is, moreover, genetically based. The genetic basis of a phenotypic correlation suggests the fundamental nature of the association, which may imply pleiotropy (i.e., one gene influences multiple traits), a circumstance in which genes that influence narcissism may also influence impulsive buying (Kovas and Plomin, 2006). This genetic correlation also suggests that genetic factors may affect narcissism and impulsive buying by influencing some common factors underlying them both. Existing evidence suggests this possibility. For instance, extraversion, neuroticism, and impulsivity are each found to be connected to both impulsive buying (Bratko et al., 2013) and the maladaptive components of narcissism (Corry et al., 2008; Hill and Roberts, 2012). Moreover, some of the connections have genetic bases (Vernon et al., 2008; 
Bratko et al., 2013). Hence, it is likely that genes influence some basic personality traits, further predisposing an individual to both narcissism and impulsive buying. Such hypotheses, however, still require additional genetic studies in the future.

Our results also showed that individual differences in narcissism and impulsive buying, together with their associations, are not entirely determined by genetic factors. Although shared environment contributes little, non-shared environment reveals substantial differences. This pattern is consistent with a large body of extant findings obtained from twin studies in personality and social psychology (Plomin et al., 2008). Individuals, including identical twins living in the same environment, differ in the ways they perceive and understand their environment, and therefore are influenced differently based on a particular environment (Hanscombe et al., 2010). Given the harmful consequences of impulsive buying, the pronounced impact of non-shared environment suggests the possibility of reducing the tendency for impulsive buying.

A series of theories and empirical findings have characterized narcissists as consumers who care more about the symbolic value than the utilitarian value of a product (Sedikides et al., 2007; Cisek et al., 2008; Lee et al., 2013). Our present studies have identified another feature of narcissistic consumers where they tend to buy on impulse. The unrestricted desire for material possessions as well as the preference for exclusive products may leave narcissists at financial risk in the long run. This possibility calls for the development of intervention strategies targeting narcissistic consumption. Since impulsive purchases contribute to almost half of daily human purchases (Nichols et al., 2001), being aware of a high impulsive buying tendency among high narcissists may be good for business promotion. Marketing practices may especially be designed to target these specific groups. To do so, of course, we need to differentiate adaptive narcissism from maladaptive narcissism.

Notably, the measures for adaptive and maladaptive narcissism only manifested moderate reliabilities. The low reliability, though consistent with previous findings (Barry et al., 2007; Barry and Malkin, 2010; Hepper et al., 2014), may reduce the power of detecting genetic influence because genetic analysis is based on correlations. Nevertheless, we still identified significant heritability for maladaptive narcissism as

\section{References}

Ackerman, R. A., Witt, E. A., Donnellan, M. B., Trzesniewski, K. H., Robins, R. W., and Kashy, D. A. (2011). What does the narcissistic personality inventory really measure? Assessment 18, 67-87. doi: 10.1177/10731911103 82845

Akaike, H. (1987). Factor-analysis and AIC. Psychometrika 52, 317-332. doi: 10.1007/BF02294359

Allport, G. W. (1937). Personality: A Psychological Interpretation. New York, NY: Holt.

Applebaum, W. (1951). Studing consumer behavior in retails stores. J. Mark. 16, 172-178. doi: 10.2307/1247625

Barry, C. T., Grafeman, S. J., Adler, K. K., and Pickard, J. D. (2007). The relations among narcissism, self-esteem, and delinquency in a sample of at-risk adolescents. J. Adolesc. 30, 933-942. doi: 10.1016/j.adolescence.2006.12.003 well as its association with impulsive buying, which suggests the potency of genetic influence on the one hand, and on the other hand, cautions that future replication is needed. As for the measure of impulsive buying, it manifested relatively high reliability, being comparable to the original study that constructed the scale (Rook and Fisher, 1995). Nevertheless, we should exercise caution recognizing that the scale might not reflect the complexity of impulsive buying. In addition, we also must be aware of the correlational nature of our present research. Although personality theories typically hypothesize traits as causes of behaviors, rather than the reverse (Allport, 1937; McCrae and Costa, 1999), future studies employing longitudinal design are needed to test possible causal relationships. Finally, as we have operationalized impulsive buying as the tendency to buy on impulse, one should keep in mind that a trait tendency may not necessarily result in real behavior (Rook and Fisher, 1995).

In summary, both phenotypic and genetic evidence suggested a connection between narcissism and impulsive buying. Being aware of the increasing tendency toward narcissism (of course including maladaptive narcissism) among young adults worldwide (Twenge et al., 2008; Cai et al., 2012), whether or not impulsive buying is on the rise constitutes an important topic for future research.

\section{Author Contributions}

HC and XF conceived and designed the experiments. YS and YL collected the data. YL analyzed the data. HC and YL drafted the paper. YS and XF provided critical revisions. All authors approved the final version of the manuscript and agreed to account for all aspects of the work.

\section{Acknowledgments}

This research was supported by the National Natural Science Foundation of China [31300871] and the Major Project of the National Social Science Foundation of China [13\&ZD073]. We gratefully acknowledge twins in BeTwiSt.

Barry, C. T., and Malkin, M. L. (2010). The relation between adolescent narcissism and internalizing problems depends on the conceptualization of narcissism. J. Res. Pers. 44, 684-690. doi: 10.1016/j.jrp.2010.09. 001

Baumeister, R. F. (2002). Yielding to temptation: self-control failure, impulsive purchasing, and consumer behavior. J. Consum. Res. 28, 670-676. doi: $10.1086 / 338209$

Boker, S. M., Neale, M. C., Maes, H. H., Wilde, M. J., Spiegel, M., Brick, T. R., et al. (2012). OpenMx 1.2 User Guide. Available at: http://openmx.psyc. virginia.edu

Bollen, K. A. (1989). Structural Equations with Latent Variables. New York, NY: John Wiley and Sons. doi: 10.1002/9781118619179

Bratko, D., Butkovic, A., and Bosnjak, M. (2013). Twin study of impulsive buying and its overlapwith personality. J. Individ. Differ. 34, 8-14. doi: 10.1027/16140001/a000091 
Cai, H., Kwan, V. S. Y., and Sedikides, C. (2012). A sociocultural approach to narcissism: the case of modern china. Eur. J. Personal. 26, 529-535. doi: 10.1002/per.852

Cash, T. F., and Cash, D. W. (1982). Women's use of cosmetics: psychosocial correlates and consequences. Int. J. Cosmet. Sci. 4, 1-14. doi: 10.1111/j.14672494.1982.tb00295.x

Chen, J., Li, X., Zhang, J., Natsuaki, M. N., Leve, L. D., Harold, G. T., et al. (2013). The Beijing Twin Study (BeTwiSt): a longitudinal study of child and adolescent development. Twin Res. Hum. Genet. 16, 91-97. doi: 10.1017/thg. 2012.115

Christopher, A. N., and Schlenker, B. R. (2000). The impact of perceived material wealth and perceiver personality on first impressions. J. Econ. Psychol. 21, 1-19. doi: 10.1016/S0167-4870(99)00033-1

Cisek, S. Z., Hart, C. M., and Sedikides, C. (2008). Do narcissists use material possessions as a primary buffer against pain? Psychol. Inq. 19, 205-207. doi: $10.1080 / 10478400802608848$

Corry, N., Merritt, R. D., Mrug, S., and Pamp, B. (2008). The factor structure of the Narcissistic Personality Inventory. J. Pers. Assess. 90, 593-600. doi: $10.1080 / 00223890802388590$

Dittmar, H., Beattie, J., and Friese, S. (1995). Gender identity and material symbols: objects and decision considerations in impulse purchases. J. Econ. Psychol. 16, 491-511. doi: 10.1016/0167-4870(95)00023-H

Dittmar, H., Beattie, J., and Friese, S. (1996). Objects, decision considerations and self-image in men's and women's impulse purchases. Acta Psychol. 93, 187-206. doi: 10.1016/0001-6918(96)00019-4

Dittmar, H., and Drury, J. (2000). Self-image-is it in the bag? A qualitative comparison between "ordinary" and "excessive" consumers. J. Econ. Psychol. 21, 109-142. doi: 10.1016/S0167-4870(99)00039-2

Emmons, R. A. (1984). Factor analysis and construct validity of the Narcissistic Personality Inventory. J. Pers. Assess. 48, 291-300. doi: 10.1207/s15327752jpa4803_11

Emmons, R. A. (1987). Narcissism: theory and measurement. J. Pers. Soc. Psychol. 52, 11-17. doi: 10.1037/0022-3514.52.1.11

Hanscombe, K. B., Haworth, C. M. A., Davis, O. S. P., Jaffee, S. R., and Plomin, R. (2010). The nature (and nurture) of children's perceptions of family chaos. Learn. Individ. Differ. 20, 549-553. doi: 10.1016/j.lindif.2010. 06.005

Hepper, E. G., Hart, C. M., Meek, R., Cisek, S. Z., and Sedikides, C. (2013). Narcissism and empathy in young offenders and non-offenders. Eur. J. Personal. 28, 201-210. doi: 10.1002/per.1939

Hepper, E. G., Hart, C. M., and Sedikides, C. (2014). Moving Narcissus: can narcissists be empathic? Pers. Soc. Psychol. Bull. 40, 1079-1091. doi: $10.1177 / 0146167214535812$

Hill, P., and Roberts, B. W. (2012). Narcissism, well-being, and observer-rated personality across the lifespan. Soc. Psychol. Personal. Sci. 3, 216-225. doi: $10.1177 / 1948550611415867$

Kacen, J. J., and Lee, J. A. (2002). The influence of culture on consumer impulsive buying behavior. J. Consum. Psychol. 12, 163-176. doi: 10.1207/S15327663JCP1202_08

Kasser, T., and Ryan, R. M. (1996). Further examining American dream: differential correlates of intrinsic and extrinsic goals. Pers. Soc. Psychol. Bull. 22, 280-287. doi: $10.1177 / 0146167296223006$

Kline, R. B. (1998). Principles and Practice of Structural Equation Modeling. New York, NY: Guilford.

Kovas, Y., and Plomin, R. (2006). Generalist genes: implications for the cognitive sciences. Trends Cogn. Sci. 10, 198-203. doi: 10.1016/j.tics.2006.03.001

Lee, S. Y., Gregg, A. P., and Park, S. H. (2013). The person in the purchase: narcissistic consumers prefer products that positively distinguish them. J. Pers. Soc. Psychol. 105, 335-352. doi: 10.1037/a0032703

Loehlin, J. C. (1996). The Cholesky approach: a cautionary note. Behav. Genet. 26, 65-69. doi: 10.1007/BF02361160

Lucas, M., and Koff, E. (2014). The role of impulsivity and of self-perceived attractiveness in impulse buying in women. Pers. Individ. Dif. 56, 111-115. doi: 10.1016/j.paid.2013.08.032

Luo, Y. L. L., Cai, H., Sedikides, C., and Song, H. (2014). Distinguishing communal narcissism from agentic narcissism: a behavior genetics analysis on the agency-communion model of narcissism. J. Res. Pers. 49, 52-58. doi: 10.1016/j.jrp.2014.01.001
Martin, N. G., Eaves, L. J., Kearsey, M. J., and Davies, P. (1978). The power of the classical twin study. Heredity (Edinb.) 40, 97-116. doi: 10.1038/hdy.1978.10

McCrae, R. R., and Costa, P. T. Jr. (1999). "A five-factor theory of personality," in Handbook of Personality: Theory and Research, 2nd Edn, eds L. A. Pervin and O. P. John (New York, NY: Guildford Press), 139-153.

McGue, M., and Bouchard, T. J. (1984). Adjustment of twin data for the effects of age and sex. Behav. Genet. 14, 325-343. doi: 10.1007/BF01080045

Miller, J. D., Campbell, W. K., Young, D. L., Lakey, C. E., Reidy, D. E., Zeichner, A., et al. (2009). Examining the relations among narcissism, impulsivity, and self-defeating behaviors. J. Pers. 77, 761-794. doi: 10.1111/j.1467-6494.2009. 00564.x

Mogelonsky, M. (1998). Keep candy in the aisles. Am. Demogr. 20, 32.

Morf, C. C., and Rhodewalt, F. (2001). Unraveling the paradoxes of narcissism: a dynamic self-regulatory processing model. Psychol. Inq. 12, 177-196. doi: 10.1207/S15327965PLI1204_1

Neale, M. C., and Cardon, L. R. (1992). Methodology for Genetic Studies of Twins and Families. Dordrecht: Kluwer. doi: 10.1007/978-94-015-8018-2

Nichols, J., Li, F., Roslow, S., Kranendonk, C. J., and Mandakovic, T. (2001). InterAmerican perspectives from mall shoppers: chile-United States. J. Glob. Mark. 15, 87-103. doi: 10.1300/J042v15n01_06

O'Guinn, T., and Faber, R. (1989). Compulsive buying: a phenomenological exploration. J. Consum. Res. 16, 147-171. doi: 10.1086/209204

Plomin, R., DeFries, J. C., McCleann, G. E., and McGuffin, P. (2008). Behavioral Genetics, 5th Edn. New York, NY: Worth Publishers.

Raskin, R. N., and Terry, H. (1988). A principal-components analysis of the Narcissistic Personality Inventory and further evidence of its construct validity. J. Pers. Soc. Psychol. 54, 890-902. doi: 10.1037/0022-3514.54.5.890

R Development Core Team. (2012). R: A Language and Environment for Statistical Computing. Vienna: R Foundation for Statistical Computing.

Ridgway, N. M., Kukar-Kinney, M., and Monroe, K. B. (2008). An expanded conceptualization and a new measure of compulsive buying. J. Consum. Res. 35, 622-639. doi: 10.1086/591108

Roberts, B. W., and Robins, R. W. (2000). Broad dispositions, broad aspirations: the intersection of the Big Five dimensions and major life goals. Pers. Soc. Psychol. Bull. 26, 1284-1296. doi: 10.1177/0146167200262009

Rook, D. W. (1987). The buying impulse. J. Consum. Res. 14, 189-199. doi: $10.1086 / 209105$

Rook, D. W., and Fisher, R. J. (1995). Normative influences on impulsive buying behavior. J. Consum. Res. 22, 305-313. doi: 10.1086/209452

Rook, D. W., and Hoch, S. J. (1985). "Consuming Impulses," in Advances in Consumer Research, eds M. B. Holbrook and E. C. Hirschman (Provo, UT: Association for Consumer Research), 23-27.

Rose, P. (2007). Mediators of the association between narcissism and compulsive buying: the roles of materialism and impulse control. Psychol. Addict. Behav. 21, 576-581. doi: 10.1037/0893-164X.21.4.576

Sedikides, C., Gregg, A. P., Cisek, S. Z., and Hart, C. M. (2007). The I that buys: narcissists as consumers. J. Consum. Psychol. 17, 254-257. doi: 10.1016/S10577408(07)70035-9

Silvera, D. H., Lavack, A. M., and Kropp, F. (2008). Impulse buying: the role of affect, social influence, and subjective wellbeing. J. Consum. Mark. 25, 23-33. doi: 10.1108/07363760810845381

Stern, H. (1962). The significance of impulse buying today. J. Mark. 26, 59-62. doi: $10.2307 / 1248439$

Twenge, J. M., Konrath, S., Foster, J. D., Campbell, W. K., and Bushman, B. J. (2008). Egos inflating over time: a cross-temporal meta-analysis of the narcissistic personality inventory. J. Pers. 76, 875-901. doi: 10.1111/j.14676494.2008.00507.x

Underhill, P. (1999). Why We Buy: The Science of Shopping. New York, NY: Simon and Schuster.

Vazire, S., and Funder, D. C. (2006). Impulsivity and the self-defeating behavior of narcissists. Pers. Soc. Psychol. Rev. 10, 154-165. doi: $10.1207 /$ s15327957pspr1002_4

Vernon, P. A., Villani, V. C., Vickers, L. C., and Harris, J. A. (2008). A behavioral genetic investigation of the Dark Triad and the Big 5. Pers. Individ. Dif. 44, 445-452. doi: 10.1016/j.paid.2007.09.007

Verplanken, B., and Herabadi, A. G. (2001). Individual differences in impulse buying tendency: feeling and no thinking. Eur. J. Personal. 15, S71-S83. doi: $10.1002 /$ per.423 
Verplanken, B., Herabadi, A. G., Perry, J. A., and Silvera, D. H. (2005). Consumer style and health: the role of impulsive buying in unhealthy eating. Psychol. Health 20, 429-441. doi: 10.1080/088704404123313 37084

Vohs, K. D., and Faber, R. J. (2007). Self-regulatory resource availability affects impulse buying. J. Consum. Res. 33, 537-547. doi: 10.1086/510228

Washburn, J. J., McMahon, S. D., King, C. A., Reinecke, M. A., and Silver, C. (2004). Narcissistic features in young adolescents: relations to aggression and internalizing symptoms. J. Youth Adolesc. 33, 247-260. doi: 10.1023/B:JOYO.0000025323.94929.d9

Watson, P., and Biderman, M. D. (1993). Narcissistic personality inventory factors, splitting, and self-consciousness. J. Pers. Assess. 61, 41-57. doi: 10.1207/s15327752jpa6101_4

Wood, N. Y., and Ahuvia, A. C. (1998). Personal taste and family face: luxury consumption in confucian and western societies. Psychol. Mark.
15, 423-441. doi: 10.1002/(SICI)1520-6793(199808)15:5<423::AID-MAR2>3.0. $\mathrm{CO} ; 2-9$

Zhang, Y., and Shrum, L. J. (2009). The influence of self-construal on impulsive consumption. J. Consum. Res. 35, 838-850. doi: 10.1086/593687

Conflict of Interest Statement: The authors declare that the research was conducted in the absence of any commercial or financial relationships that could be construed as a potential conflict of interest.

Copyright (c) 2015 Cai, Shi, Fang and Luo. This is an open-access article distributed under the terms of the Creative Commons Attribution License (CC BY). The use, distribution or reproduction in other forums is permitted, provided the original author(s) or licensor are credited and that the original publication in this journal is cited, in accordance with accepted academic practice. No use, distribution or reproduction is permitted which does not comply with these terms. 